\title{
Solar radius in 2005 and 2006 eclipses
}

\section{Costantino Sigismondi}

ICRA,International Center for Relativistic Astrophysics,P.le Aldo Moro 500185 Rome, Italy E-mail: sigismondi@icra.it

\begin{abstract}
Baily beads timings of annular eclipse of October 3, 2005 and total eclipse of March 29, 2006 have been analyzed for measuring a variation of the actual solar radius between those dates. After $1 / 3$ of all beads events analyzed, corrections to average solar radius are known within $\pm 0.19 "$ ". High resolution video have been made at the boundaries of totality-annularity paths, to increase the number of beads visible. Comparison of observations made with different combinations of telescopes/filters/detectors is discussed.
\end{abstract}

Keywords. Eclipses, Techniques: high angular resolution, Methods Data Analysis

Introduction. The analysis of historical eclipses data: 1715 (Halley, 1717); 1925 (Sofia et al., 1980; Thuillier et al., 2005) and recent high resolution videorecords in the period 1984-2002 (Fiala et al. 1994; Dunham et al. 2005) already indicated solar radius variations up to 0.5 " with respect to mean value of 961.63 " at unit distance. In 1567 annular eclipse, observed in Rome by Clavius, solar radius should be $\sim+2.5$ " larger, if the ring he observed at maximum eclipse was not the inner corona as retained by Stephenson et al. (1997). Geometrical circumstances of Baily beads are determined out of the atmosphere; the seeing does not affect the timing of appearence or disappearence of a bead. Merging and divisions of beads, meanwhile, undergo instrumental black drop effect (Schneider et al., 2001), therefore we not use them. This method is the only one for high resolution $<0.1$ " measurements of the whole solar diameter: present and future solar imaging techniques are limited to fields of view $<1$ ' either in space or ground-based.

Observations. In Zawyet al Mahtallah, Egypt, P. Colona (PC, thanks) and myself (CS, $373 \mathrm{~m}$ SE of PC) observed the eclipse perpendicularly to the Southern path's edge with 2 twin telescopes Meade ETX 70 with orange photo filters Tamron Y2A, projecting images $(\varnothing>10 \mathrm{~cm})$ on white lucid screens. Those images have been registered with digital camcorders ( $>50$ beads events lasting 5 minutes over $220^{\circ}$ or $61 \%$ of lunar limb). In central location A. B. Morcos, (ABM, thanks) $136 \mathrm{~m}$ SE from PC, was on zero eclipse point for unvariated solar radius, as simulated by D. Herald's (thanks) Winoccult 3.1.ABM aimed at the Sun with unfiltered SONY digital camcorder with lens $\varnothing=2.4 \mathrm{~cm}$ and recorded 8 beads events for $1.5 \mathrm{~min}$ and $6 \mathrm{~min}$ of corona view around maximum eclipse. Totality lasted $\tau_{\mathrm{ABM}}=-4.80 \mathrm{~s}$. Closer to the edge PC registered $\tau_{\mathrm{PC}}=-1.28 \mathrm{~s}$ and myself $\tau_{\mathrm{CS}}=-4.68 \mathrm{~s}$. If last disappearing bead is visible with first appearing $\tau<0$. Location $\pm 1 \mathrm{~m}$ and timing $\pm 0.04 \mathrm{~s}$ were assured by Garmin GPS II+ receiver (thanks to G. Dusi) with a video synchronizations of internal camcorders' clocks.

Comparison between different instrumental apparatus. Direct view allowed ABM to record corona (inner and outer) and beads brighter than in telescope projected images, where the corona was not visible. To observe the same $\tau_{\mathrm{ABM}}=-4.8 \mathrm{~s}$ our projecting instruments would have been located $\sim 242 \mathrm{~m}$ SE of actual ABM position. This shift corresponds to $\mathbf{0 . 1 2}$ " at Moon's distance (perpendicular to the line of sight), and it is the $\Delta r_{\odot}$ in excess to our telescope data. Spanish observers of annular eclipse of Oct. 3, 2005 used filtered (transmittance $t=0.00025,9$ magnitudes drop) and telescopes $(\varnothing=\sim 7 \mathrm{~cm}, 2.3$ magnitude gain with respect to SONY camcorder). Since the 
limiting magnitude of SONY camcorders is $m_{\text {lim }}=2$, it becomes $m_{\text {lim }}=-4.7$ coupled with such filtered telescopes. Solar limb luminosity is $16 \%$ of the center disk value, i.e. $-8.6 \mathrm{mag} / \operatorname{arcsec}^{2}$ and only a bead area $A>1 / 36 \operatorname{arcsec}^{2}$ is visible. Solar area shown through lunar valleys $\mathrm{V}$-shaped with slopes $\alpha=5^{\circ}$ is $A=d^{2} / \operatorname{tg} \alpha$ : beads' minimum detectable depth is $d=0.05$ ". Rectangular valleys 1 " wide give $d=0.03$ ". So we expect Spanish data giving $\Delta r_{\odot} \sim 0.08$ " larger than our projected views and $\mathbf{0 . 0 4}$ " smaller than direct unfiltered views, to which hereinafter $\Delta r_{\odot}$ are referred (naked-eye corrections).

Data analysis: method and results. Winoccult 3.1 generates Watts (1963) profiles of lunar limb; it was used to identify beads events in video (Watts angle WA and height of solar limb relative to mean lunar limb). With no Morrison-Appleby (1981) corrections to Watts profiles and no shifts to lunar ephemeris, in ABM location, a variation of \pm 1 $\mathrm{s}$ in totality duration corresponds to $\Delta r_{\odot} \mp 0.033$ " in solar radius. For a bead at WA $=\alpha$ the solar limb moves at $v_{\odot, \alpha} \sim \pm 0.1$ " / s, therefore an uncertainty of 0.1 " in bead's depth can change totality duration of $\sim 2 \mathrm{~s}$, not due to radius variations. Analyzing timings of many beads we reduce statistically this uncertainty. Two and Three-parameters analysis. For each bead residuals (Sun's height - lunar limb profile) and solar limb speed $v_{\odot, \alpha}$ are computed. Residuals are corrected for each angle $\alpha$ by $\Delta T_{\odot} \times v_{\odot, \alpha}$ and $\Delta R_{\odot}$ is the average of residuals and $\Delta T_{\odot}$ minimize their standard deviation. $\Delta T_{\odot}$ corresponds to ecliptic latitude correction in lunar ephemeris, but takes also into account possible synchronization errors. Fitting the residuals with arcs centered at the WA of maximum eclipse, lunar latitude corrections $\Delta l$ are found: they vanish at WA $\pm 90^{\circ}$ from there.

Annular Eclipse of 2005, Oct. 3. Our expedition with P. Oliva (thanks) and PC in Valoria La Buena was clouded, but I received useful data of Northern limit from C. Schnabel, J. Rovira, A. Selva, J. Lopez, O. Canales $\left(^{*}\right)$ and C. Perelli (*) (thanks to all) I selected the same 9 bead events in $\left(^{*}\right)$, after including W. Strickling 6 beads (thanks) at Southern limit I obtained $\Delta R_{\odot \text { Oct. } 05}=-0.06 "+\mathbf{0 . 0 4} " \pm 0.19 "$ and $\Delta T_{\odot}=-0.1 \mathrm{~s}$, without $\Delta l$ because of beads full angular coverage. Total Eclipse of 2006, March 29. Using PC and CS data both at Southern limit, and Watts profiles without Morrison Appleby (1981) corrections $\Delta T_{\odot}$ is closer to zero. Naked-eye correction of $\Delta R_{\odot \text { Mar. } 06}=$ $-0.24 "+\mathbf{0 . 1 2} " \pm 0.16 ", \Delta T_{\odot}=-0.2 \mathrm{~s}$, which has to include a $\Delta l=0.13 "$. Furthermore I observed inner corona for $\sim 4$ minutes with naked eye, screening the Sun's crescent with the thumb when needed. Inner corona is irregular and not like a ring, with many spikes, invisible on beads side. Clavius saw a perfect ring, that only a photosphere with Sun radius $\Delta R_{\odot 1567}>+2.5$ " could produce. Such thin ring $\sim 13$ magnitudes brighter than spikes canceled their view. Conclusions. After conversion to naked-eye corrections, solar radius changes $\Delta R_{\odot}$ range from -0.02 " to -0.25 " in six months from Oct. 3,2005 to March, 29 2006. Their errorbars are still $0.16 " \div 0.19$ " at the present stage of analysis. If that reduction of solar radius will be confirmed by the whole beads dataset, this will be an evidence of radius anticorrelation with solar activity. Acknowledgments To Prof. R. Ruffini \& ICRA who sustained this research, and to all contributing observers.

\section{References}

Halley, E., 1717 Philosophical Transactions XXIX, 245-262

Watts,C.B. 1963 Marginal Zones of the Moon US Gov.Pr.Off.XVII, Washington

Sofia, S., et al. 1980 Science 210, 1243-1245

Morrison, L.V. and Appleby G.M. 1981 MNRAS 196, 1013-1020

Fiala, A. D., Dunham, D. W. and S. Sofia 1994 Solar Phys. 152, 97-104

Stephenson, F. R., et al. 1997 Astron. \& Astrophys. 322, 347-35

Schneider, G., et al. 2001 33rd meeting AAS Planetary Science New Orleans, Louisiana

Dunham, D. W., et al. 2005 SORCE Science Meeting Sept. 14-16 Durango, Colorado

Thuillier, G., Sofia, S. and M. Haberreiter, 2005 Advances in Space Res. 35 329-340 